\title{
Alcune varianti di nomi di frutti
}

\author{
Mara Marzullo
}

PUBBLICATO: 18 LUGLIO 2003

\section{Quesito:}

Claudio Sgarbanti chiede chiarimenti su alcuni termini ortofrutticoli, ovvero cocomero (o, si domanda, cocomera, anguria?), zucchina o zucchino, melone ("alcuni toscani lo chiamano popone").

\section{Alcune varianti di nomi di frutti}

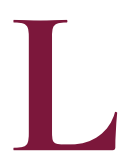

e piante e i frutti della famiglia della zucca hanno creato spesso confusioni nella storia della nostra lingua, al punto che sin nei primi vocabolari cinquecenteschi del volgare italiano la scelta di lemmatizzare popone o melone poteva essere indicativa del grado di toscanità del lessico raccolto. Non a caso Adriano Politi, un lessicografo senese dei primi del Seicento desideroso di rivendicare un ruolo al senese a fianco del fiorentino, scriveva nel suo Dittionaro toscano (I6I5): "mellone. Fiorent. frutto simile alla zucca lunga, di colore simile al cedruolo, ma più sciapito. Sen. chiamano questo frutto col nome di cedruolo. E per mellone intendono il popone".

La situazione è complessa, dunque, ed entrano in gioco, come avviene spesso per i termini legati all'alimentazione, alla cucina e in genere a usi domestici, le varianti regionali. Cerchiamo allora di fare un po' di chiarezza, anche attraverso le indicazioni dell'Atlante italo-svizzero (K. Jaberg-J. Jud, Sprachund Sachatlas Italien und der Südschweiz, 1937), nel quale si possono seguire le distribuzioni su tutto il territorio italiano di termini legati a 'zucca' (vol. VII-2, c. I372) e a 'cetriuolo' (c. I373).

Prima di tutto il caso più semplice: zucchino-zucchina? Il primo termine attestato è zucchino (I875) seguito a breve (1879) da zucchina: entrambi derivano da 'zucca' e ne sono in origine i diminutivi, uno composto sul maschile e uno sul femminile. Sarebbe preferibile il maschile: sia il Grande dizionario della lingua italiana (diretto da S. Battaglia) sia il Grande dizionario italiano dell'uso (diretto da T. De Mauro) rinviano da zucchina a zucchino e corredano solo il sostantivo maschile della definizione della pianta.

Angurie e cocomeri: anguria è variante settentrionale per il toscano cocomero; il nome entra in italiano attraverso il veneziano dal greco tardo angóuria plurale di angóurion 'cetriolo' (Mattioli, av. 577 scrive: "Chiamiamo noi Toscani le angurie, cocomeri" e Carena nel Nuovo vocabolario italiano domestico del I869 chiarisce: "Questo cucurbitaceo, il cui nome linneano è Cucumis Anguria, i Toscani chiamano Cocomero, i Lombardi Anguria, cosi i primi lo denominano dal genere, i secondi dalla specie"). E, considerando altre varietà regionali, "A Napoli il cocomero è detto melone d'acqua (melon d'eau) e melone da pane, il popone. In Calabria, zi pàrrucu (zio parroco), cioè rubicondo come il volto del parroco" (Panzini, Dizionario moderno, 1942). Il cocomero è comunque la forma panitaliana. Nello slittamento settentrionale dei termini, cocomero indica il cetriolo (sempre Panzini: "In Lombardia poi chiamano 'cocomero' (cocumer) quello che altrove si chiama 'cetriolo' e si prepara sotto aceto. Similmente a Genova").

Infine, melone e popone. Sul valore "toscanizzante" del termine nella storia della nostra lingua si è già detto all'inizio: melone è la forma di diffusione italiana e anche nel toscano il popone, che è pretto toscano, tende a essere accostato a melone. Alla varietà di legami tra nome e frutto corrisponde anche 
una diversità di forme con cui i nomi sono documentabili nelle aree della penisola: come mellone, sia per forme attestate nell'uso antico, quindi in distribuzione temporale e non spaziale, come per poppóne, pupóne, puppóne e per cocómaro, cocúmero.

Allargando, in margine, al campo semantico ai frutti si può ricordare un altro caso di uso fortemente connotato in senso fiorentino: diospero per il 'cachi'. A tal proposito Montale nell'Elegia di Pico Farnese del 1939 scriveva "diaspori", mentre l'edizione critica del r980 (a cura di Gianfranco Contini, Rosanna Bettarini, L'Opera in versi, Torino, Einaudi) restituisce "diosperi"; lo stesso poeta, d'altronde, in una lettera a Bibo Bazlen del 9 giugno 1939 con alcune indicazioni di traduzione in tedesco chiariva il verso "Se urgi fino al midollo i diosperi" così: "Se urgi (o se gonfi) ecc. i frutti del kaki."

\section{Cita come:}

Mara Marzullo, A/cune varianti di nomi di frutti, "Italiano digitale", 2003, IX, 2019/2 (aprile-giugno) DOI: $10.35948 / 2532-9006 / 2020.3158$ 